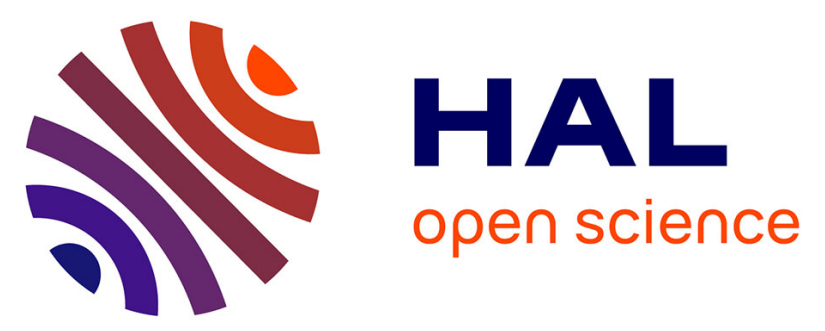

\title{
3D frequency-domain finite-difference modeling of acoustic wave propagation using a massively parallel direct solver: a feasibility study
}

Stéphane Operto, Jean Virieux, Patrick Amestoy, Luc Giraud, Jean-Yves L'Excellent

\section{To cite this version:}

Stéphane Operto, Jean Virieux, Patrick Amestoy, Luc Giraud, Jean-Yves L'Excellent. 3D frequencydomain finite-difference modeling of acoustic wave propagation using a massively parallel direct solver: a feasibility study. SEG 2006 - 76th International Exposition and Annual Meeting, Oct 2006, New Orleans, United States. pp.2265-2269, 10.1190/1.2369987 . hal-00408484

\section{HAL Id: hal-00408484 \\ https://hal.science/hal-00408484}

Submitted on 25 Jan 2022

HAL is a multi-disciplinary open access archive for the deposit and dissemination of scientific research documents, whether they are published or not. The documents may come from teaching and research institutions in France or abroad, or from public or private research centers.
L'archive ouverte pluridisciplinaire HAL, est destinée au dépôt et à la diffusion de documents scientifiques de niveau recherche, publiés ou non, émanant des établissements d'enseignement et de recherche français ou étrangers, des laboratoires publics ou privés.

\section{(c) (1) $\$$}

Distributed under a Creative Commons Attribution - NonCommerciall 4.0 International 


\title{
3D frequency-domain finite-difference modeling of acoustic wave propagation using a massively par- allel direct solver: a feasibility study
}

\author{
S. Operto *, J. Virieux, Géosciences Azur, P. Amestoy, L. Giraud ENSEEIHT-IRIT, J. Y. L'Excellent, INRIA-ENS-Lyon
}

\section{SUMMARY}

We present a frequency-domain finite-difference method for modeling $3 \mathrm{D}$ acoustic wave propagation based on a massively parallel direct solver. This method was developed as a tool for frequency-domain full-waveform inversion of 3D global offset data that requires an efficient modeling code for multiple shots and few frequencies.

We have first implemented a finite-difference stencil for the 3D acoustic frequency-domain wave equation in pressure. The method is based on the parsimonious mixed-grid formulation which linearly combines several second-order accurate staggered-grid stencils on different rotated coordinate frames to minimize the numerical anisotropy. Accuracy of the stencil is improved by using an average of the mass acceleration term at the collocation point. Our approach leads to a 27-point stencil. The resultant system of linear equations is solved with the massively parallel solver MUMPS based on a multifrontal method. We have computed multi-shot simulations in the 3D SEG/EAGE overthrust model to assess whether representative seismic imaging problems can be tackled with the frequency-domain approach. The $5 \mathrm{~Hz}$ frequency has been modeled in a portion of the overthrust model of dimension $20 \times 11.4 \times 4.5 \mathrm{~km}$ using a PC cluster of 20 bi-processor nodes with $4 \mathrm{Go}$ of memory each.

We conclude that, using larger clusters, 3D frequency-domain fullwaveform modeling and inversion applied to dense global offset acquisition geometries may provide a reliable tool for imaging the largeto middle- wavelengths of geological structures.

\section{INTRODUCTION}

Quantitative seismic imaging of 3D crustal structures is one of the main challenge of geophysical exploration at different scales (subsurface, oil exploration, crustal and lithospheric investigations). Few applications of frequency-domain full-waveform inversion applied to 2D onshore and offshore global offset seismic data have been recently presented to image complex structures such as thrust belt or subduction zone (Ravaut et al., 2004; Operto et al., 2006). The potential interest of such approaches is to exploit the full aperture range spanned by global offset geometries to image a broad and continuous range of wavelengths in the medium including large to middle wavelengths. The frequency-domain approach of full-waveform inversion has been shown to be efficient for several reasons (e.g., Pratt et al. (1998)): first, only few discrete frequencies are necessary to develop a reliable image of the medium thanks to the wavenumber redundancy provided by multi-aperture geometries. Moreover, proceeding sequentially from the low to the high frequencies defines a multiresolution imaging strategy which helps to fulfill the assumptions associated with linearized inverse methods.

In 2D, the few frequency components required to solve the inverse problem can be efficiently modeled in the frequency domain using a finite-difference (FD) method. Modeling of one frequency with a finite-difference method requires to solve a large and sparse system of linear equations. If this system can be solved with a direct solver, the solution for multiple right-hand side terms (i.e., multiple sources) can be obtained very efficiently which is crucial for tomographic applications. Indeed, the factorization of the matrix is solved once and the multiple solutions can be rapidly obtained by forward and backward substitutions.

The drawback of the direct approach compared to the iterative counterpart is the huge core memory required by the fill-in of the factored matrix (the matrix after LU factorization). Todays, modern computers with shared or distributed memory allow to tackle the 2D frequencydomain full-waveform modeling and inversion problems for representative imaging problems at the oil exploration and deep crustal scales (Ravaut et al., 2004; Operto et al., 2006). The aim of this study is to assess the feasibility of 3D frequency-domain finite-difference modeling of wave propagation based on a massively parallel direct solver MUMPS (MUMPS-team, 2006; Amestoy et al., 2006). We first present the FD stencils that have been developed for the 3D frequencydomain finite-difference (FDFD) wave equation. Second, we present some simulations in the 3D SEG/EAGE overthrust model performed with a small PC cluster composed of 20 bi-processor nodes.

\section{DISCRETIZATION OF THE 3D WAVE EQUATION}

The method is the $3 \mathrm{D}$ extension of the $2 \mathrm{D}$ parsimonious mixed-grid method (Hustedt et al., 2004). The wave equation is first written as a first-order hyperbolic system in pressure-particle velocities in the frequency-domain,

$$
\begin{aligned}
& \frac{-i \omega}{K(x, y, z)} P(x, y, z, \omega)=\frac{1 .}{\xi_{x}(x)} \frac{\partial V_{x}(x, y, z, \omega)}{\partial x}+\frac{1}{\xi_{y}(y)} \frac{\partial V_{y}(x, y, z, \omega)}{\partial y}+\frac{1}{\xi_{z}(z)} \frac{\partial V_{z}(x, y, z, \omega)}{\partial z} \\
& =S(x, y, z, \omega) \\
& V_{x}(x, y, z, \omega)=\frac{i b(x, y, z)}{\omega \xi_{x}(x)} \frac{\partial P(x, y, z, \omega)}{\partial x} \\
& V_{y}(x, y, z, \omega)=\frac{i b(x, y, z)}{\omega \xi_{y}(y)} \frac{\partial P(x, y, z, \omega)}{\partial y} . \\
& V_{z}(x, y, z, \omega)=\frac{i b(x, y, z)}{\omega \xi_{z}(z)} \frac{\partial P(x, y, z, \omega)}{\partial z} .
\end{aligned}
$$

where $P, V_{x}$ and $V_{z}$ are the pressure and particle-velocity wavefields respectively. $S$ is the explosive source. $K$ and $b$ are bulk modulus and buoyancy respectively. $\omega$ is angular frequency. $\xi_{i}=1+j \gamma_{i} / \omega$ are PML functions controlling the wave absorption on all four sides of the model and $\gamma_{i}$ are damping functions in the PML layers ( $\mathrm{i}=\mathrm{x}, \mathrm{y}$ and $\mathrm{z}$ and $j^{2}=-1$ ) (Hustedt et al., 2004).

The first-order spatial derivatives in equation 1 are discretized using second-order accurate centered staggered-grid stencils on different rotated coordinate frames (e.g., Virieux (1984); Saenger et al. (2000)). Second-order accurate staggered-grid stencils are used because their local spatial extent limits the bandwidth of the impedance matrix to be factorized (Jo et al., 1996). Different rotated coordinate frames are defined for discretization. Their axis must cover as many directions as possible in order to limit the numerical anisotropy. We have used three main coordinate frames consistent with the staggered-grid geometry: [1] the classic cartesian one (Figure 1a). [2] one obtained by rotation around one cartesian axis (Figure 1b). This defines 3 basis by rotation around $x, y$ and $z$ respectively, and therefore, 3 elementary FD stencils. [3] one formed by the 4 big diagonal of a cubic cell (Figure 1c). This defines 4 additional basis and, therefore, 4 additional stencils (Saenger et al., 2000). Note that these coordinate frames differ from those introduced by Stekl and Pain (2002) who proposed to use, in addition to the coordinate frames [1] and [2], one coordinate frame obtained by rotation around 2 of the Cartesian axis. This defines 6 additional stencils which are not consistent with a staggered-grid geometry in the sense that they would require to define more than one pressure grid.

Once the equation system 1 has been discretized with the 7 staggeredgrid FD stencils, particle velocities are eliminated from the system leading to 7 discrete second-order wave equation in pressure. After elimination, only the pressure wavefield remains but the underlying 
staggered-grid structure still appears in the parsimonious formulation through the estimation of the buoyancy parameter at intermediate positions with respect to the pressure grid points. The parsimonious strategy provides a systematic recipe to discretize second-order wave equation from its first-order representation. After particle-velocity elimination, the 3 discrete wave equations of the coordinate frame [2] can be averaged as well as the 4 equations of the coordinate frame [3]. We end up with 3 discrete second-order wave equations associated with the coordinate frames [1], [2] and [3] which define a 9-point, 19-point and 27point stencils respectively. The three discrete wave equations can be linearly combined to minimize phase velocity dispersion. Moreover, the mass term at the collocation point is replaced by its average over the grid points involved in the 27-point stencil to improve the accuracy of the stencil (Jo et al., 1996).

The wave equation discretized with the parsimonious mixed-grid strategy can be compactly written as

$$
\left\{\left[\sum_{l=1}^{4} w m_{l} \mathscr{M}_{l}(x, y, z, \omega)+\sum_{n=1}^{3} w_{n} \mathscr{S}_{n}(x, y, z, \omega)\right] P(x, y, z, \omega)\right\}_{i, j, k}=S_{i, j, k}
$$

where $\mathscr{M}$ and $\mathscr{S}$ denote the discrete mass and stiffness operators respectively. $w$ denotes the weights applied to the 3 discrete wave equations associated with each coordinate frame. These weights verify $w_{1}+w_{2}+w_{3}=1$.

For the mass acceleration term, we have

$$
\begin{aligned}
& {\left[\mathscr{M}_{1}\right]_{i, j, k}=\frac{\omega^{2}}{K_{i, j, k}}} \\
& {\left[\mathscr{M}_{2}\right]_{i, j, k}=\frac{\omega^{2}}{K_{i \pm 1, j, k}}+\frac{\omega^{2}}{K_{i, j \pm 1, k}}+\frac{\omega^{2}}{K_{i, j, k \pm 1}}} \\
& {\left[\mathscr{M}_{3}\right]_{i, j, k}=\frac{\omega^{2}}{K_{i \pm 1, j \pm 1, k}}+\frac{\omega^{2}}{K_{i \pm 1, j, k \pm 1}}+\frac{\omega^{2}}{K_{i, j \pm 1, k \pm 1}}} \\
& {\left[\mathscr{M}_{4}\right]_{i, j, k}=\frac{\omega^{2}}{K_{i \pm 1, j \pm 1, k \pm 1}}}
\end{aligned}
$$

where we have used the summation convention, $\frac{\omega^{2}}{K_{i \pm 1, j, k}}=\frac{\omega^{2}}{K_{i+1, j, k}}+$ $\frac{\omega^{2}}{K_{i-1, j, k}}$. The weights $w m$ verify $w m_{1}+w m_{2} / 6+w m_{3} / 12+w m_{4} / 8=$ 1 .

The coefficients $w$ and $w m$ are computed by optimization such that the phase velocity dispersion is minimized for different directions of propagation. We used a simple grid search algorithm to find a first estimate of the solution followed by a Gauss-Newtown local optimization. The normalized phase velocity as a function of the number of grid points per wavelength, $G$, is shown in Figure 2. The optimization was computed for $G=4$.

The pattern of the impedance matrix discretized with the 27-point stencil is shown in Figure 3 for a small 3D grid of dimensions $8 \times 8 \times 8$. The order of the matrix is $8^{3}$. The matrix is band diagonal with fringes. The bandwidth of the matrix is $\mathscr{O}\left(n_{1} n_{2}\right)$ where $n_{1}$ and $n_{2}$ are the two smallest dimensions of the $3 \mathrm{D}$ grid. The number of non zero coefficients per row is 27 .

To solve the system 2, we have used the massively parallel direct solver MUMPS which is based on a multifrontal method and distributed dynamic scheduling of tasks (Amestoy et al., 2006; MUMPSteam, 2006). The algorithm is subdivided into 3 main phases: [i] an analysis phase which computes a reordering of the matrix coefficients that will reduce the fill-in of the factored matrix and a symbolic factorization. For the re-ordering phase we have used the algorithm METIS (Karypis and Kumar, 1998); [ii] the factorization based on a multifrontal method. The reader is referred to (e.g., Liu (1992)) for a review on the multifrontal method; [iii] the resolution for multiple shots.

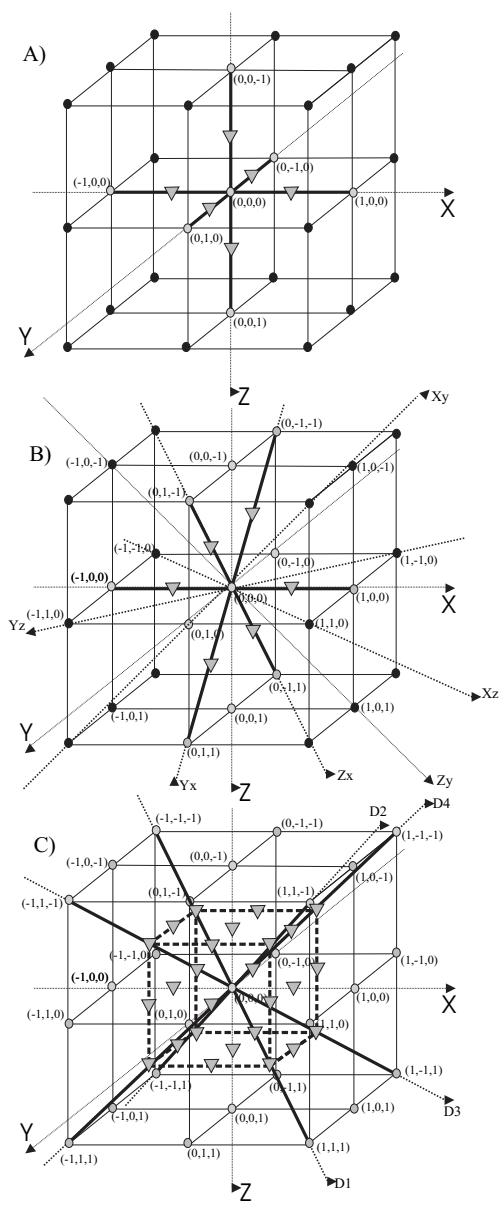

Figure 1: Coordinate frames used for discretization of spatial derivatives. An elementary cubic cell is plotted. The circles mark the reference pressure grid. Gray circles are the grid points involved in the stencil. The triangles indicate the locations of the buoyancy required to compute the spatial derivatives. The thick black lines indicate the direction along which spatial derivatives are discretized. a) Cartesian frame. b) Coordinate frame obtained by rotation around the $\mathrm{x}$ - axis. Similar rotation around $y$ and $z$ are also used leading to a 19-point stencil. c) Coordinate frames formed by the 4 big diagonals leading to a 27-point stencil.

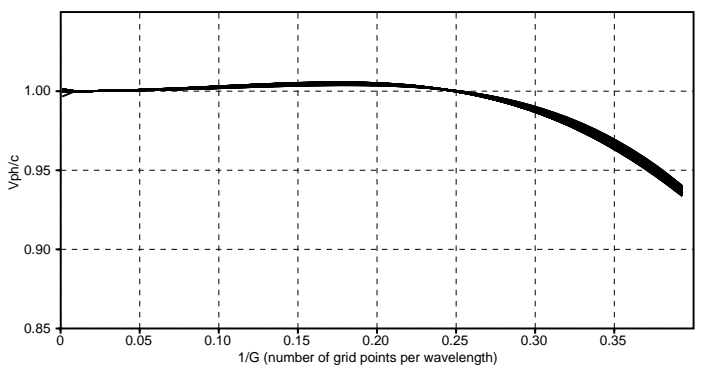

Figure 2: Dispersion curves for different directions of plane-wave propagation in a homogeneous medium. The direction of propagation of the plane wave is defined by 2 angles which vary between 0 and $45^{\circ}$ in the figure. 


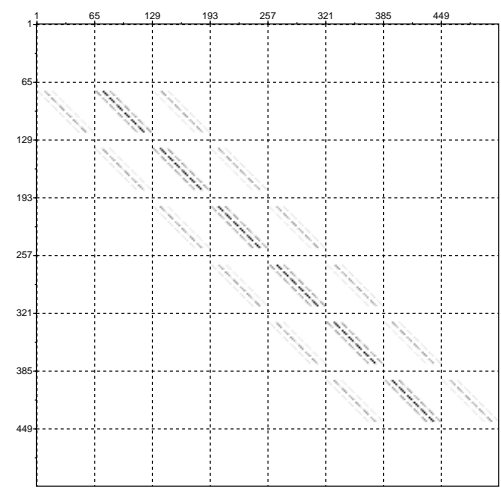

Figure 3: Impedance matrix for the 27-point stencil and a $8^{3}$ grid.

The accuracy of the 27-point stencil was verified by comparing [i] the 3D FDFD solution with the analytical one for a homogeneous medium (Figure 4), [ii] the 2D FDFD solution computed in a corner-edge velocity model (Hustedt et al., 2004) with the 3D FDFD one. The 3D solution has been computed using a line source in a 2.5D extension of the 2D model to mimic a 2D simulation (Figure 5).

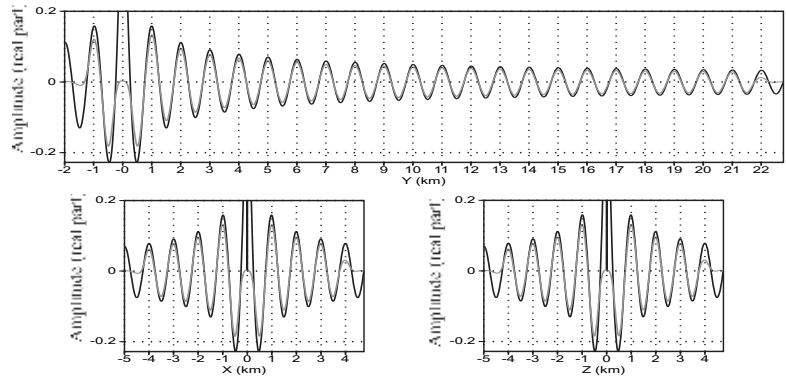

Figure 4: Comparison between graphs extracted from 4-Hz wavefields computed analytically (black) and by FDFD (gray) in a $4 \mathrm{~km} / \mathrm{s}$ homogeneous medium. Graphs have been extracted along $x, y$ and $z$. The horizontal axis are source-receiver offset. The agreement is good except near the zero-offset singularity of the analytical solution.

\section{APPLICATION TO THE 3D SEG/EAGE OVERTHRUST MODEL}

To assess the feasibility of 3D FDFD modeling of acoustic wave propagation for realistic problems, we have computed simulations in the 3D SEG/EAGE overthrust model for a realistic 3D global-offset acquisition geometry. The 3-D SEG/EAGE Overthrust model is a constant density acoustic model covering an area of $20 \mathrm{~km} \times 20 \mathrm{~km}$ x $4.65 \mathrm{~km}$ (Aminzadeh et al., 1995). It is discretized with $25 \mathrm{~m}$ cubic cells, representing an uniform mesh of $801 \times 801 \times 187$ nodes. The dominant frequency of the simulated seismic experiment is about $15 \mathrm{~Hz}$.

For our simulations, we have used a small PC cluster of 20 bi-processor nodes with 4Go of memory per node. Two processes were allocated per node. We have first considered the $5 \mathrm{~Hz}$ frequency. This leads to a grid interval of $100 \mathrm{~m}$ to verify the sampling condition of 4 grid points per minimum wavelength. The overthrust model has been smoothed with a Gaussian filter of horizontal and vertical correlation lengths of $150 \mathrm{~m}$ and undersampled with a mesh interval of $100 \mathrm{~m}$. With such grid interval, we have been able to compute simulations in a fraction of the overthrust model of dimension $20 \times 10.7 \times 4.6 \mathrm{~km}$ (Figure 6 ). This corresponds to a 3D rectangular grid of dimension $208 \times 116 \times 55$
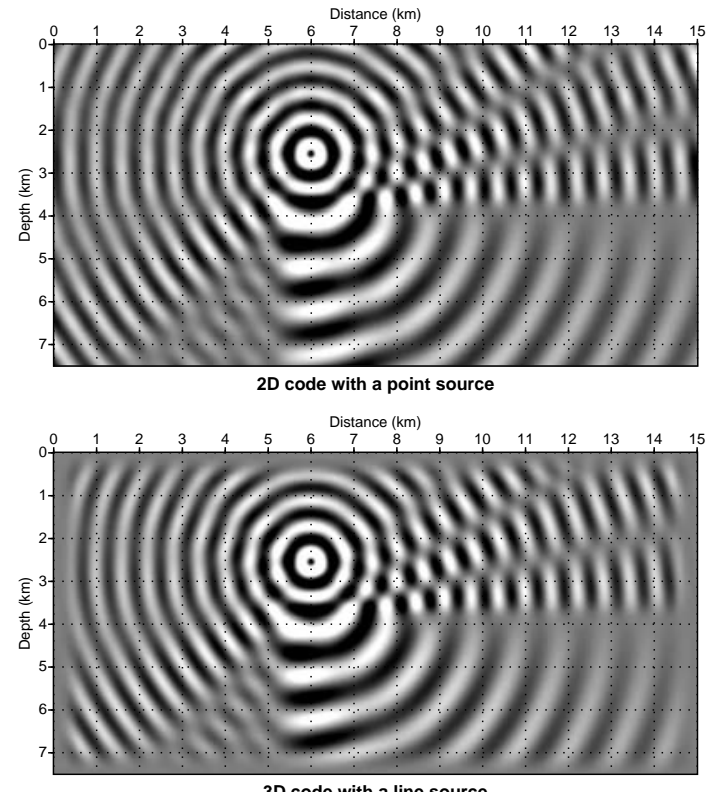

Figure 5: Corner-edge model. Top: solution computed with a 2D code. Bottom: solution computed with a 3D code. The plot of the 3D solution includes the absorbing PML layers on contrary to the 2D one. Frequency is $5 \mathrm{~Hz}$. P-wave velocities are $3 \mathrm{~km} / \mathrm{s}$ and $5 \mathrm{~km} / \mathrm{s}$ in the upper-left and lower-right layers respectively. The corner is at $(5.2,3.6) \mathrm{km}$. The source is at $(6,2.5) \mathrm{km}$.

which incorporates 4 grid points in the PML layers. We have computed simulations for 420 sources representing an array of land stations with a spacing of $1 \mathrm{~km}$ and $500 \mathrm{~m}$ along $\mathrm{x}$ - and $\mathrm{y}$ - respectively (thanks to reciprocity of Green's functions, receivers can be processed as shots in the frame of full-waveform inversion).

The total memory that has been allocated during factorization is around 70 Go. The elapsed time for factorization and resolution for all the 420 sources is around $83 \mathrm{mn}$ and $42 \mathrm{mn}$ respectively leading to a total elapsed time of around 2 hours to complete the 420 simulations (Table 1). Two examples of mono-frequency wavefields are shown in Figure 6(c-d). A wavepath, that is the sensitivity kernel of frequencydomain full-waveform inversion, has been computed for a short-offset source-receiver pair. The width of the isochrones gives some insights on the vertical resolution which would be achieved by full-waveform inversion at $5 \mathrm{~Hz}$. This resolution is of the order of $200 \mathrm{~m}$ and $500 \mathrm{~m}$ near the surface and the bottom of the model respectively.

\section{CONCLUSIONS AND FUTURE WORKS}

These preliminary results suggest that representative seismic imaging problems could be tackled using large cluster with up to 1.6 To of distributed memory (considering 400 processors with 4 Go of memory each). With such an amount of memory, simulations may be computed in the full overthrust model for frequencies up to $8 \mathrm{~Hz}$ (assuming a memory requirement of $\mathscr{O}\left(n^{5}\right)$ ). At these frequencies, yet rather accurate images of the overthrust model can be obtained (Figure 7 for an illustration in the 2D case). Future works will concern [i] the use of out-of-core memory in the factorization, [ii] the combined use of direct and iterative solvers, [iii] the improvement of multiple-shot resolution (out-of-core and parallelization) and, the [iii] the implementation of a 3D frequency-domain full-waveform inversion algorithm. 

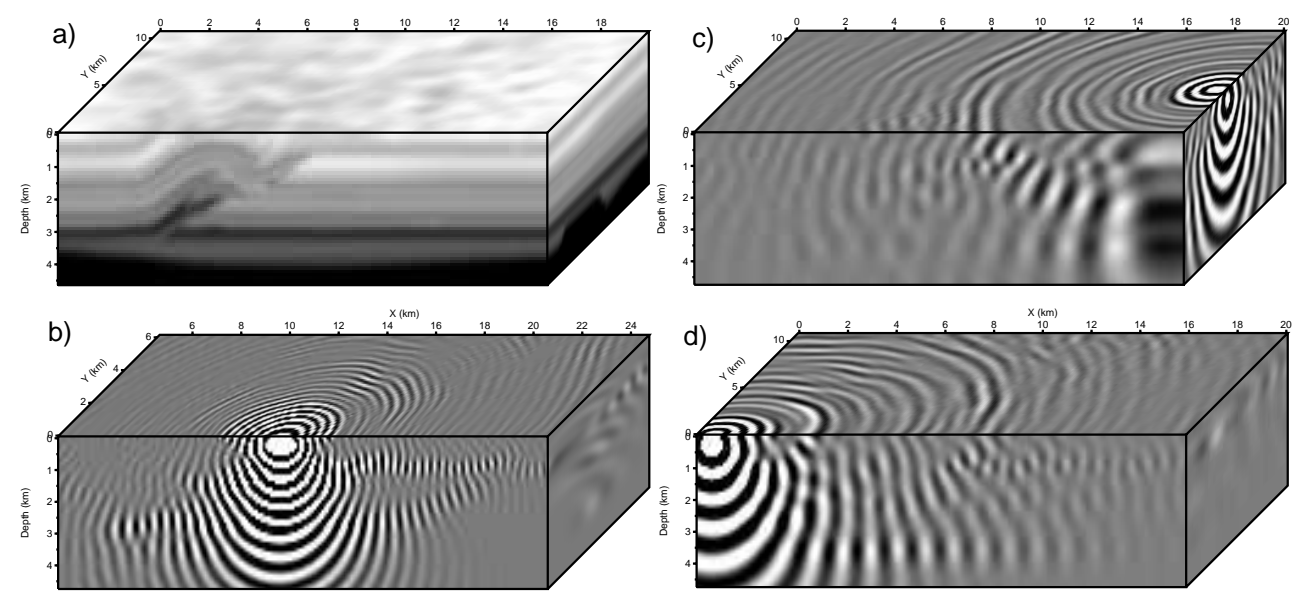

Figure 6: a) 3D overthrust model after smoothing with a 2D Gaussian filter of correlation lengths $150 \mathrm{~m}$. b) Example of wavepath for short-offset source-receiver pair. The width of the isochrones gives some insights on the expected vertical resolution of full-waveform inversion for the $5 \mathrm{~Hz}$ frequency. (c-d) Examples of $5 \mathrm{~Hz}$ monochromatic pressure wavefields.

\begin{tabular}{|c|c|c|c|c|c|c|}
\hline \multicolumn{7}{|c|}{ Computational cost of the overthrust simulation } \\
\hline$n_{x}$ & $n_{y}$ & $n_{z}$ & $M E M_{\text {tot }}$ & $M E M_{\text {proc }}$ & ETF & ETR \\
\hline \hline 208 & 116 & 55 & 71474 & 2232 & 4994 & 5.7 \\
208 & 116 & 55 & 69976 & 2166 & 5101 & 8.8 \\
\hline
\end{tabular}

Table 1: $\left(n_{x}, n_{y}, n_{z}\right)$ : dimensions of the 3D velocity grid. $M E M_{t o t}$ : Total memory allocated during factorization. $M E M_{\text {proc }}$ : Maximum memory allocated to one processor. ETF: elapsed time for factorization. ETR: Elapsed time for resolution per shot. Two simulations have been computed with (first line) and without (second line) involving the master processor in the factorization.

\section{ACKNOWLEDGMENTS}

This work was carried out in the frame of the SEISCOPE consortium sponsored by BP, CGG, TOTAL and SHELL.

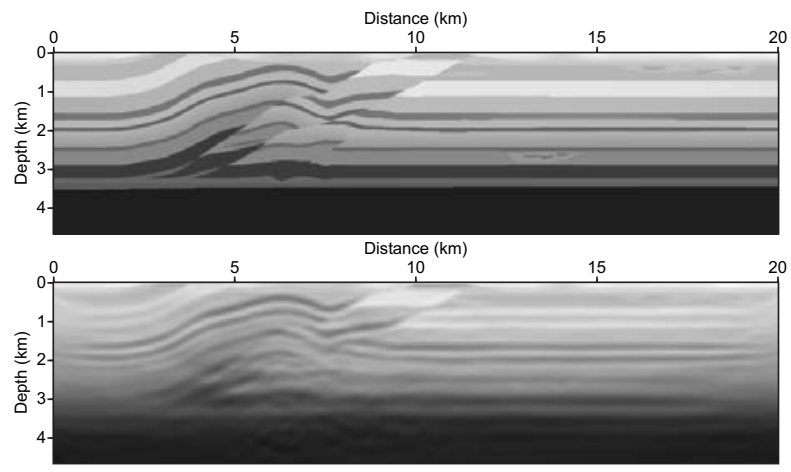

Figure 7: Top: dip section of the Overthrust model. Bottom: Velocity model obtained by $2 \mathrm{D}$ frequency-domain full-waveform inversion close of the $8 \mathrm{~Hz}$ frequency inversion.

\section{REFERENCES}

Amestoy, P. R., A. Guermouche, J. Y. L'Excellent, and S. Pralet, 2006, Hybrid scheduling for the parallel solution of linear systems: Par-

allel computing, 32, 136-156.

Aminzadeh, F., N. Burehard, L. Nicoletis, F. Rocca, and K. Wyatt, 1995, SEG/EAEG 3-D modeling project : Third report: The Leading Edge, 14, 125-128.

Hustedt, B., S. Operto, and J. Virieux, 2004, A direct-iterative solver for seismic wave propagation modelling: a space and wavelet approach: Geophysical Journal International, 155, 953-980.

Jo, C.-H., C. Shin, and J. H. Suh, 1996, An optimal 9-point, finitedifference, frequency-space, 2-d scalar wave extrapolator: Geophysics, 61, 529-537.

Karypis, G. and V. Kumar, 1998, METIS - a software package for partitioning unstructured graphs, partitioning meshes and computing fill-reducing orderings of sparse matrices - version 4.0. University of Minnesota.

Liu, J. W. H., 1992, The multifrontal method for sparse matrix solution: theory and practice: SIAM review, 34, 82-109.

MUMPS-team, 2006, MUMPS - MUltifrontal Massively Parallel Solver users' guide - version 4.6.1 (february 2006). ENSEEIHT-ENS Lyon, http://www.enseeiht.fr/apo/MUMPS/ or http://graal.ens-lyon.fr/MUMPS.

Operto, S., J. Virieux, and J. X. Dessa, 2006, Frequency-domain fullwaveform inversion of OBS wide-angle seismic data: 68th Annual EAGE Conference and Exhibition, Expanded Abstracts, in-press.

Pratt, R. G., C. Shin, and G. J. Hicks, 1998, Gauss-newton and full newton methods in frequency-space seismic waveform inversion: Geophys. J. Int., 133, 341-362.

Ravaut, C., S. Operto, L. Improta, J. Virieux, A. Herrero, and P. Dell'Aversana, 2004, Multiscale imaging of complex structures from multifold wide-aperture seismic data by frequency-domain full-waveform tomography: application to a thrust belt: Geophysical Journal International, 159, 1032-1056.

Saenger, E. H., N. Gold, and A. Shapiro, 2000, Modeling the propagation of elastic waves using a modified finite-difference grid: Wave motion, 31, 77-92.

Stekl, I. and C. Pain, 2002, 3D frequency domain visco-acoustic modeling using rotated finite difference operators: EAGE 64th Conference and exhibition, Abstract book, C-27.

Virieux, J., 1984, SH wave propagation in heterogeneous media, velocity-stress finite difference method: Geophysics, 49, 12591266. 


\section{EDITED REFERENCES}

Note: This reference list is a copy-edited version of the reference list submitted by the author. Reference lists for the 2006 SEG Technical Program Expanded Abstracts have been copy edited so that references provided with the online metadata for each paper will achieve a high degree of linking to cited sources that appear on the Web.

\section{REFERENCES}

Amestoy, P. R., A. Guermouche, J. Y. L'Excellent, and S. Pralet, 2006, Hybrid scheduling for the parallel solution of linear systems: Parallel Computing, 32, $136-156$.

Aminzadeh, F., N. Burehard, L. Nicoletis, F. Rocca, and K. Wyatt, 1995, SEG/EAEG 3D modeling project : Third report: The Leading Edge, 14, 125-128.

Hustedt, B., S. Operto, and J. Virieux, 2004, A direct-iterative solver for seismic wave propagation modelling: a space and wavelet approach: Geophysical Journal International, 155, 953-980.

Jo, C.-H., C. Shin, and J. H. Suh, 1996, An optimal 9-point, finitedifference, frequencyspace, 2-d scalar wave extrapolator: Geophysics, 61, 529-537.

Karypis, G., and V. Kumar, 1998, METIS - a software package for partitioning unstructured graphs, partitioning meshes and computing fill-reducing orderings of sparse matrices - version 4.0: University of Minnesota.

Liu, J. W. H., 1992, The multifrontal method for sparse matrix solution: theory and practice: SIAM review, 34, 82-109.

MUMPS-team, 2006, MUMPS - MUltifrontal Massively Parallel Solver users' guide version 4.6.1: ENSEEIHT-ENS Lyon, http://www.enseeiht.fr/apo/MUMPS/ or http://graal.ens-lyon.fr/MUMPS.

Operto, S., J. Virieux, and J. X. Dessa, 2006, Frequency-domain fullwaveform inversion of OBS wide-angle seismic data: Presented at the 68th Annual Conference and Exhibition, EAGE.

Pratt, R. G., C. Shin, and G. J. Hicks, 1998, Gauss-newton and full newton methods in frequency-space seismic waveform inversion: Geophysical Journal International, 133, 341-362.

Ravaut, C., S. Operto, L. Improta, J. Virieux, A. Herrero, and P. Dell'Aversana, 2004, Multiscale imaging of complex structures from multifold wide-aperture seismic data by frequency-domain full-waveform tomography: application to a thrust belt: Geophysical Journal International, 159, 1032-1056.

Saenger, E. H., N. Gold, and A. Shapiro, 2000, Modeling the propagation of elastic waves using a modified finite-difference grid: Wave Motion, 31, 77-92.

Stekl, I., and C. Pain, 2002, 3D frequency domain visco-acoustic modeling using rotated finite difference operators: $64^{\text {th }}$ Annual Conference and Exhibition, EAGE, Extended Abstracts, C-27.

Virieux, J., 1984, SH wave propagation in heterogeneous media, velocity-stress finite difference method: Geophysics, 49, 1259-1266. 